\title{
Physical examination tests for hip dysfunction and injury
}

\author{
Michael P Reiman, Richard C Mather III, Chad E Cook
}

Correspondence to Dr Michael P Reiman, Department of Community and Family Practice, Duke University School of Medicine, 2200 W. Main, Durham NC 27705, USA;michael.reiman@duke.edu

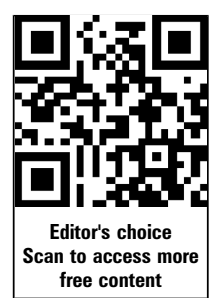

CrossMark

To cite: Reiman MP, Mather III RC, Cook CE. $\mathrm{Br} J$ Sports Med 2015;49: 357-361.
ABSTRACT

Background Physical examination tests for hip dysfunction and injury of the strongest diagnostic accuracy were identified in a recent systematic review with meta-analysis in BJSM. These tests are described in this article.

Discussion A detailed description of the various different tests is given, with photographs for each test procedure. Diagnostic interpretation of each test requires careful consideration, with special attention to specific variables such as test performance and patient population.

\section{INTRODUCTION}

Diagnosis of non-arthritic hip pathology is challenging. In the last 10 years the understanding of hip pathology and femoroacetabular impingement (FAI) has exploded, ${ }^{1}$ although in the spectrum of medicine knowledge this area is relatively new. ${ }^{2}$ As such, expertise is often located in irregular pockets of providers, resulting in variable understanding and recognition in the larger medical community. Patients with intra-articular hip pathology have been reported to see 3.3 providers on average before being correctly diagnosed. ${ }^{34}$ Thus, it is not uncommon for a patient to undergo inappropriate imaging and, unfortunately, inappropriate treatments including epidural steroid injections, hernia repairs and even lumbar spine fusion. Furthermore, long-standing hip pain results in surrounding dysfunction of the low back, pelvis and even knee, with poorer outcomes in patients with concomitant conditions. ${ }^{5}$ These factors converge to make diagnosis of hip pathology one of the greatest challenges currently facing the orthopaedic and sports medicine field.

Disorders of the hip can largely be categorised as intra-articular or extra-articular. Intra-articular disorders are driven by pathology at opposite ends of the morphological spectrum; acetabular dysplasia or FAI. ${ }^{3}{ }^{6}$ Labral tears are recognised to be the pain generator in these disease states. Extra-articular disorders include psoas tendon disorders such as internal snapping hip and tendinitis, peritrochanteric space disorders such as external snapping hip and abductor tendon tears, and deep gluteal space syndrome, previously known as piriformis syndrome. ${ }^{7}$

Whereas imaging is clearly important for correct diagnosis, false positives with MRI and MRI with arthrogram (MRA) are common, requiring providers to determine whether a distinct pathology is actually symptomatic, further relying on accurate and efficient physical examination. Solving this problem requires two primary approaches: (1) identify the most accurate-specific hip physical examination (HPE) tests and (2) provide detailed descriptions and instructions to providers to allow reproduction and application of these tests. The first was recently addressed by Reiman $e t$ al ${ }^{8}$ in a systematic review and meta-analysis. This article encompassed a detailed execution of the HPE tests with the greatest clinical utility as outlined by that group. In addition, the publication discussed the discrepancy in test description and reason for caution in test interpretation when appropriate.

\section{PHYSICAL EXAMINATION TESTS OF THE HIP Hip osteoarthritis \\ Trendelenburg's sign ${ }^{9}$}

Patient position: Standing in front of the examiner, with both feet on ground.

Clinician position: Observing the patient from the front.

Movement: The patient is instructed to lift one leg up by flexing their hip and knee, standing on only one leg (figure 1A).

Assessment: The clinician assesses the weightbearing leg by evaluating the degree of drop of the contralateral pelvis once the leg is lifted. A pelvic on femoral angle with $\leq 83^{\circ}$ angle criteria with specified time duration of $30 \mathrm{~s}$ was used as a positive sign. Figure $1 \mathrm{~B}$ shows the pelvic on femoral angle (angle between the two lines).

Diagnostic accuracy: Sensitivity (SN) 55\%, specificity (SP) $70 \%$, positive likelihood ratio (+LR) 1.83 and negative likelihood ratio (-LR) 0.82 .

Special note: Monitor for patient compensating by leaning their trunk to avoid having pelvis drop. Leaning compensation constitutes a positive test as well.

Background: Generally considered a physical performance test of hip strength, this test has also been utilised for assessment of gluteal tendinopathy with a positive test being reproduction of spontaneous pain within $30 \mathrm{~s}$ on involved leg compared with the contralateral leg during single leg stance. ${ }^{10}$

The use of a supporting stick was suggested in the hand only on the side of the weight-bearing hip. Alternatively, both shoulders could be supported by the examiner so as to maintain balance without a stick. ${ }^{11}$

\section{Commentary on hip osteoarthritis tests}

The Trendelenburg's sign alters post-test probability of a diagnosis to a very small degree. ${ }^{8}$ The clinician should carefully incorporate functional assessment (gait, stairs, etc) as part of the examination continuum even though they have not been specifically investigated for this cohort. In addition, consideration of additional components such as (1) more 
(a)

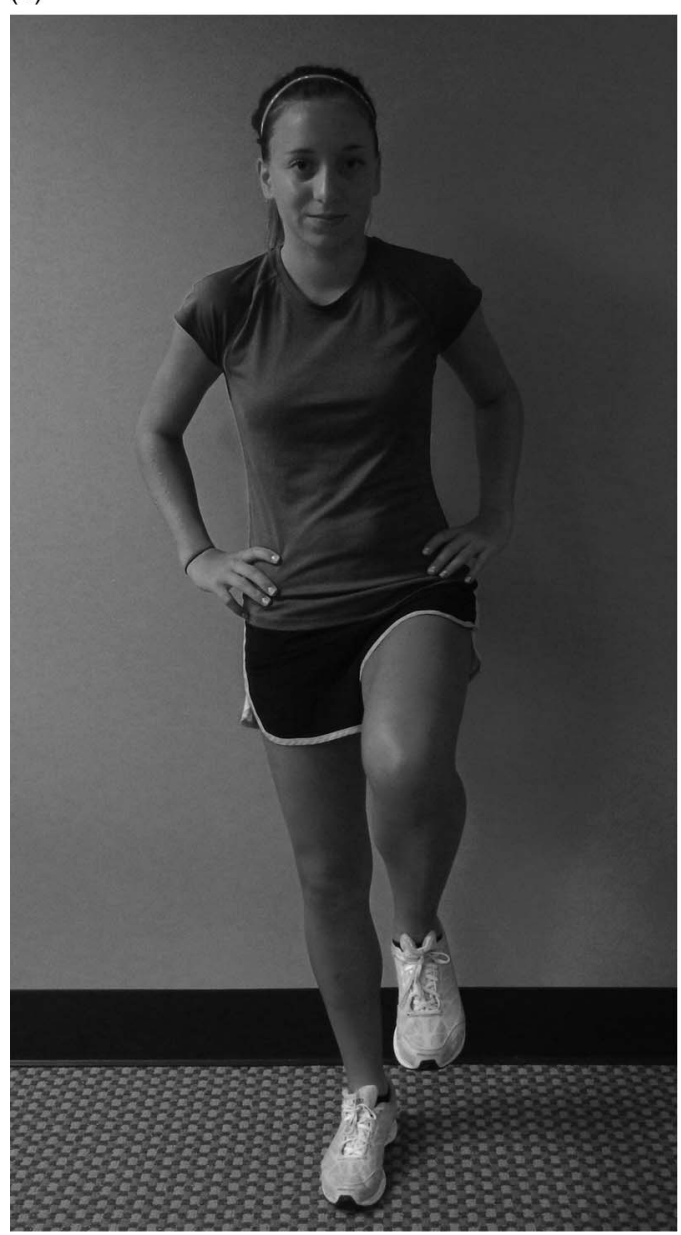

(b)

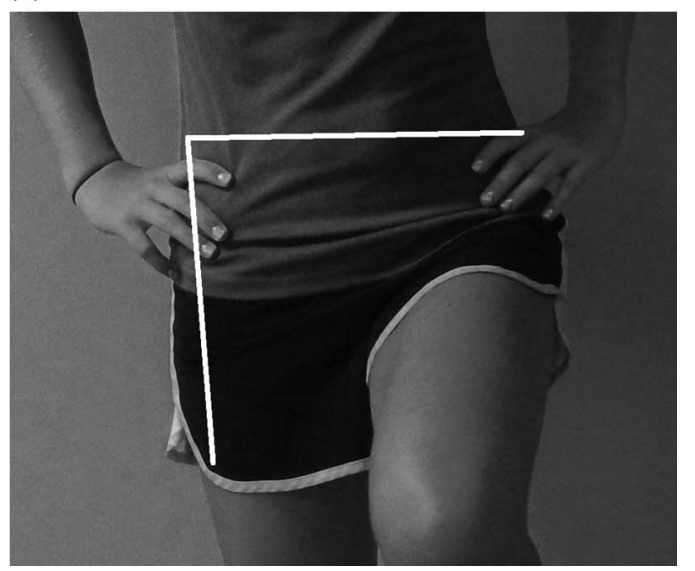

Figure 1 (A) Trendelenburg's sign. (B) Angle of measurement for Trendelenburg's sign.

than one plane of motion restriction, (2) age $>50$ and (3) stiffness $\leq 60 \mathrm{~min}$, are a necessity for clinical assessment of hip osteoarthritis. $^{12} 13$

\section{Gluteal tendinopathy}

Resisted external derotation test

Patient position: Supine, hip flexed $90^{\circ}$, and in external rotation. $^{10}$

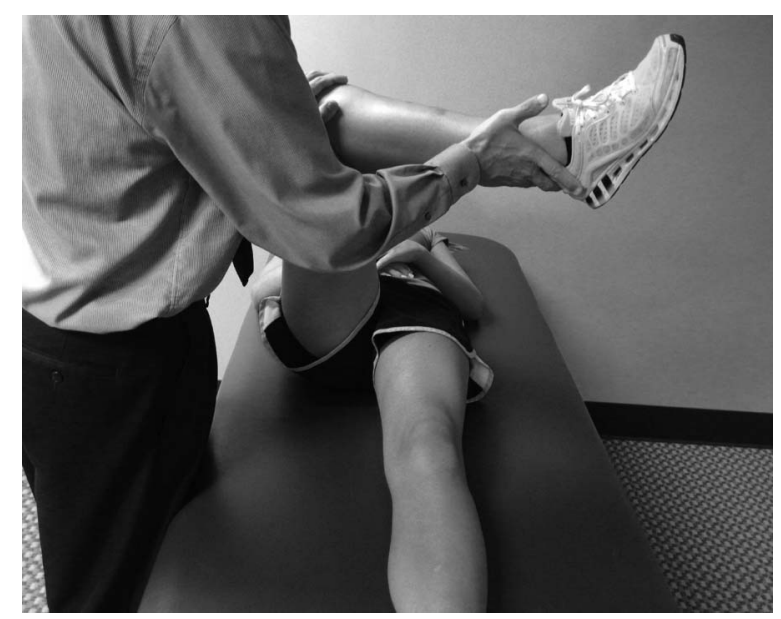

Figure 2 Resisted external derotation test.

Clinician position: Clinician, standing just to the side of leg being tested, slightly decreases external rotation just enough to relieve pain (if any was present).

Movement: Patient then actively returns the leg to neutral position (placing leg along the axis of the bed) against resistance (figure 2).

If test result is negative, the test is repeated with patient lying prone, hip extended and knee flexed to $90^{\circ}$.

Assessment: A positive test is spontaneous reproduction of patient's concordant pain.

Diagnostic accuracy: SN 88\%, SP 97.3\%, +LR 32.6 and -LR 0.12 .

Special note: Monitor for patient compensation of grabbing onto table for stabilisation during test.

Background: The combination of passive stretch, followed by active contraction is likely to provide the tensile load across the involved structure(s).

Commentary on gluteal tendinopathy tests

Only the resisted external derotation test demonstrated the ability to modify the post-test probability of a gluteal tendinopathy diagnosis. ${ }^{8}$ The sole study examining this test only had a sample size of 17 participants, with an average age of 68.1 \pm 10.8 years, thus limiting the external generalisability. The Trendelenburg's sign (as described above in the Hip osteoarthritis section) demonstrated a pooled SN of $61 \%$, SP $92 \%$, +LR 6.83 and -LR of $0.25 ;^{8}$ across three studies with 78 patients. $^{101415}$

\section{Impingement/labral tear/intra-articular pathology}

Impingement (flexion-adduction-internal rotation) (FADDIR) test Patient position: Supine, bilateral legs extended. ${ }^{16-20}$

Clinician position: Standing at the side of the leg to be tested.

Movement: Clinician passively moves the patient's leg to $90^{\circ}$ of hip and knee flexion. The leg is then passively adducted and internally rotated with overpressure to both motions at endrange (figure 3 ).

Assessment: A positive test is reproduction of concordant pain, locking, clicking and catching.

Pooled diagnostic accuracy:

(MRA criterion reference) SN 94\%, SP 8\%, +LR 1.02, -LR $0.48 ;^{8}$ across four studies with 128 patients. ${ }^{16} 171920$

(Arthroscopy criterion reference) SN 99\%, SP 7\%, +LR 1.06, -LR $0.15 ;^{8}$ across two studies with 157 patients. ${ }^{18} 19$ 


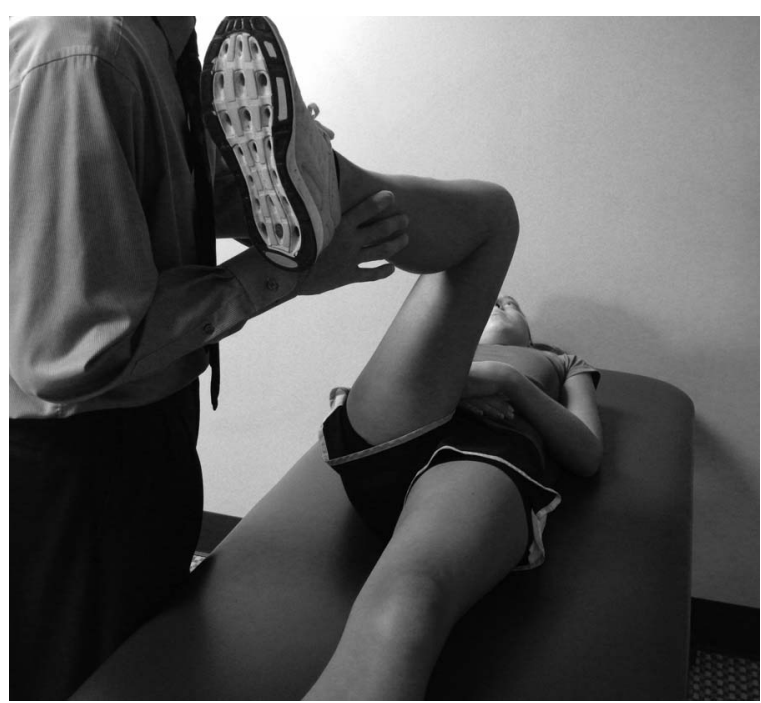

Figure 3 Flexion-adduction-internal rotation test.

Special note: Monitor for patient compensation of rolling trunk toward non-involved leg to avoid pain. Discordant lateral hip pain is a negative test.

Background: The combination motions of flexion, adduction and internal rotation cause an abutment between the femoral head and anterior acetabulum.

\section{Flexion-internal rotation test}

Patient position: Supine, bilateral legs extended. ${ }^{21-23}$

Clinician position: Standing at the side of the leg to be tested.

Movement: Clinician passively performs the combined movements of flexion to $90^{\circ}$ and internal rotation (figure 4).

Assessment: A positive test is reproduction of concordant pain, locking, clicking or catching.

Pooled diagnostic accuracy: SN 96\%, SP 17\%, +LR 1.12, -LR $0.27 ;^{8}$ across three studies with 42 patients. $^{21-23}$

Special note: Monitor for patient compensation of rolling trunk toward non-involved leg to avoid pain.

Background: This test produces a likely similar abutment described for the FADDIR test without the end-range adduction. In addition, moving from neutral adduction to end-range internal rotation could impinge an anterior labral tear.

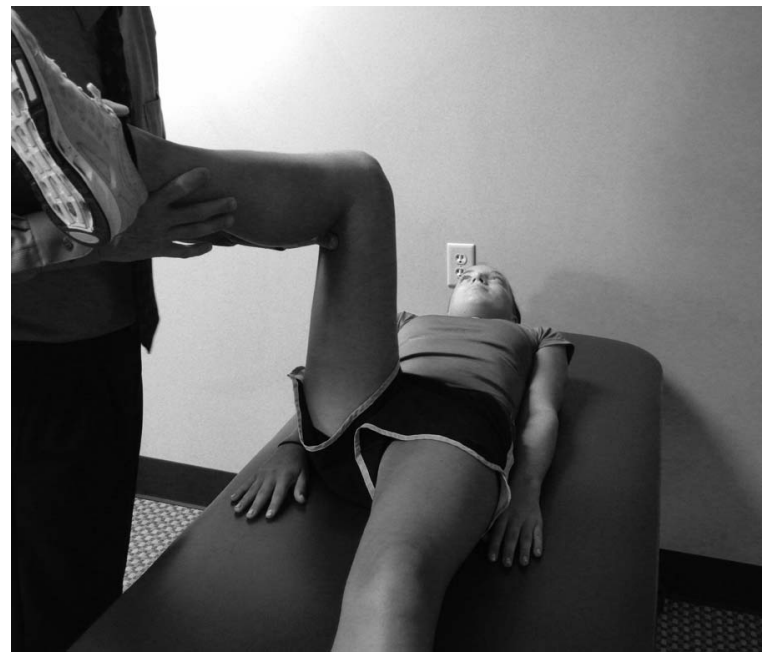

Figure 4 Flexion-internal rotation test.
Thomas test

Patient position: Sitting at the end of the table, feet on floor. ${ }^{24}$

Clinician position: Standing at end of table, directly facing patient.

Movement: Clinician passively lays the patient onto their back, bringing bilateral knees up to patient's chest. Patient holds non-tested leg toward their chest with bilateral arms as the clinician passively lowers the tested leg into extension. The clinician stabilises the ipsilateral side of the pelvis with their other arm (figure 5).

Assessment: A positive test is reproduction of painful click or concordant groin pain.

Diagnostic accuracy: SN 89\%, SP 92\%, +LR 11.1 and -LR $0.12 .^{24}$

Special note: Monitor for tightness/compensation of the

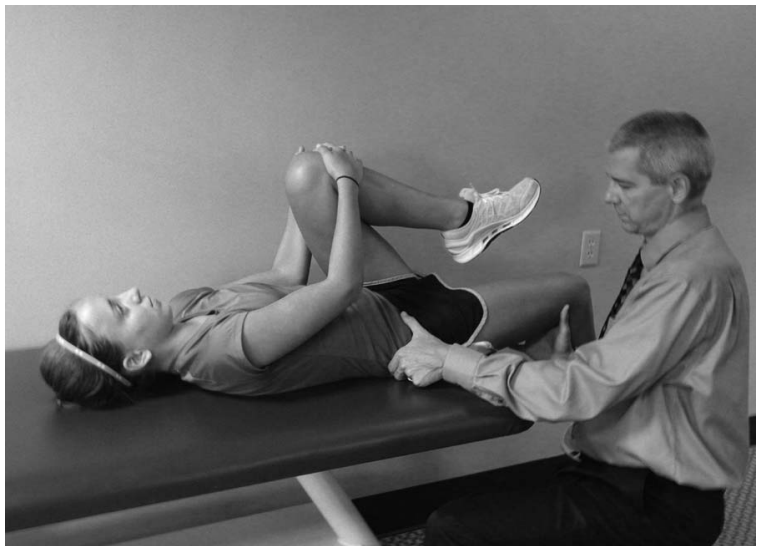

Figure 5 Thomas test.

lumbar spine arching, tested leg abducting and externally rotating.

Background: Although this test does not reproduce the mechanical abutment between the femoral head and acetabulum similar to the FADDIR or flexion internal rotation test, it does recreate hip extension, which has been shown to recreate the greatest forces on the hip joint. ${ }^{25}$

Commentary on impingement/labral tear/intra-articular pathology tests

In general, these tests demonstrate better screening than diagnostic ability. ${ }^{8}$ The one study with the least risk of bias demonstrated that the Thomas test has value as both a screen and diagnostic test. ${ }^{24}$ Caution should be used though as this was only one study. ${ }^{24}$

\section{Femoral fracture/stress fracture}

Patellar-pubic percussion test

Patient position: Supine, bilateral legs extended. ${ }^{26-28}$

Clinician position: Standing at the side of the leg to be tested.

Movement: Clinician places a stethoscope over the pubic tubercle of the patient. Clinician taps the patella of patient's leg being assessed and qualitatively reports the sound. A tuning fork has also been used in place of tapping (figure 6).

Assessment: A positive test is diminished percussion noted compared with contralateral side.

Pooled diagnostic accuracy: SN 95\%, SP 86\%, +LR 6.11, -LR $0.07 ;^{8}$ across three studies with 782 patients. ${ }^{26-28}$ 


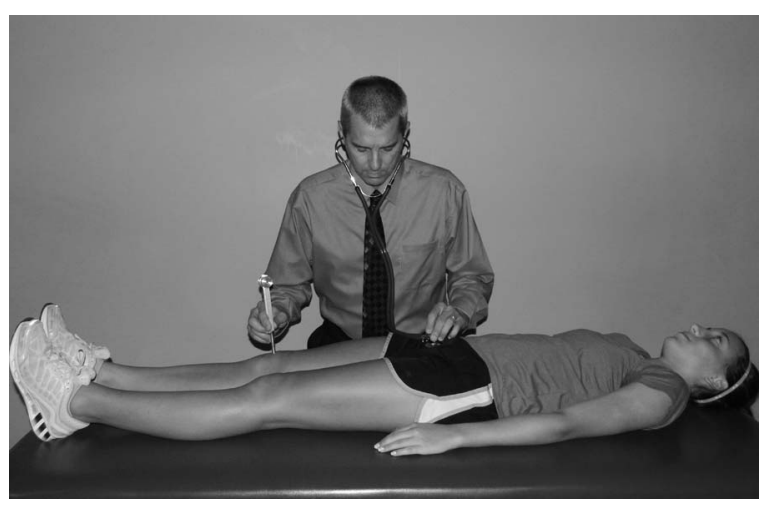

Figure 6 Patellar-pubic percussion test.

Special note: Clinician must ensure that stethoscope is placed firmly over pubic tubercle and lateral to the pubic symphysis joint (on the side ipsilateral to side being tested).

Background: The sound produced with either tapping or the tuning fork is dampened with the fracture/stress fracture.

\section{Fulcrum test}

Patient position: Sitting on side of table with bilateral distal portion of legs off the edge of the table. ${ }^{29}$ Patient is instructed to lean back on bilateral hands.

Clinician position: Standing or kneeling to the side of the leg to be tested.

Movement: Clinician places one forearm under patient's thigh to be tested. Clinician arm is used as a fulcrum under the thigh and is moved from the distal to the proximal thigh as gentle pressure is applied to the dorsum of the knee with the opposite arm (figure 7).

Assessment: A positive test is reproduction of patient's concordant discomfort/sharp pain, usually accompanied by apprehension.

Diagnostic accuracy: SN 93\%, SP 75\%, +LR 3.7, -LR $0.09 ;^{29}$ SN $88 \%$, SP $13 \%$, +LR 1.0, -LR 0.92..$^{30}$

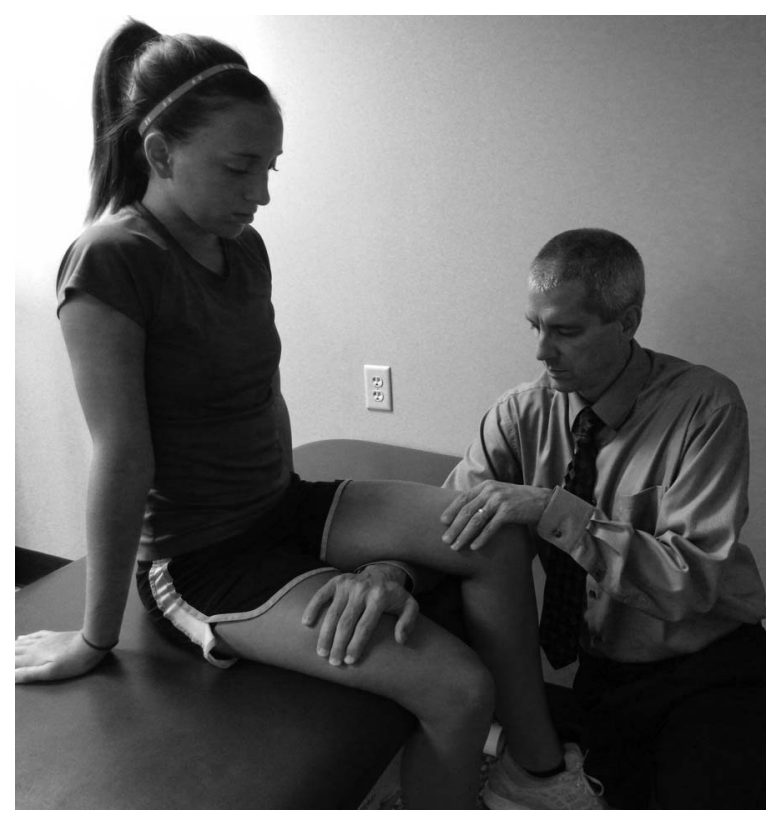

Figure 7 Fulcrum test.

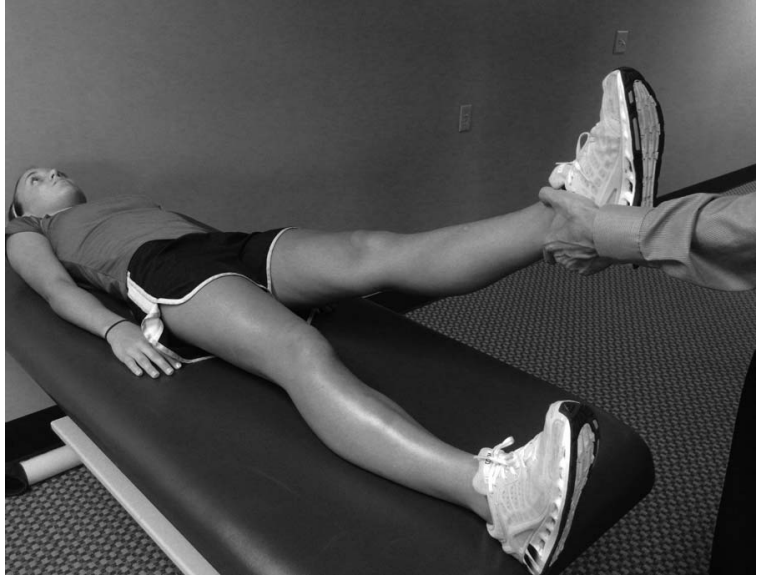

Figure 8 Single adductor test.

Special note: The length of the femur assessed is limited due to the ability to place the arm under the assessed femur.

Background: Fulcrum and pressure applied in the direction opposite creates stress force to the area of suspected stress fracture.

Commentary on fracture/stress fracture tests

The patellar-pubic percussion test has strong diagnostic value as both a screen and diagnostic test. ${ }^{8}$ The use of stethoscope and tuning fork has previously been demonstrated as a valid measure for this diagnosis. ${ }^{31}$ Caution is suggested with the use of the stress fracture test (despite demonstrated ability to function as a screening test) due to high risk bias and small subject sizes in studies investigating this test.

\section{Sports related chronic groin pain}

Single adductor test

Patient position: Patient is supine with bilateral legs extended. ${ }^{32}$

Clinician position: Standing at patient's foot to be assessed.

Movement: Clinician passively flexes leg to be assessed to $30^{\circ}$ with slight abduction and internal rotation. Patient resists the clinicians attempt to abduct the leg to be tested, effectively contracting their adductor muscles on that side (figure 8).

Assessment: A positive test involves reproduction of patient's concordant pain.

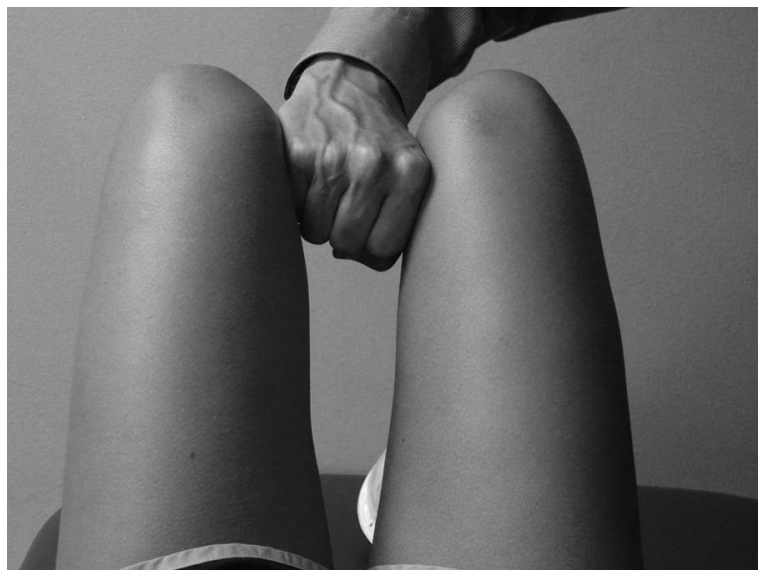

Figure 9 Squeeze test. 


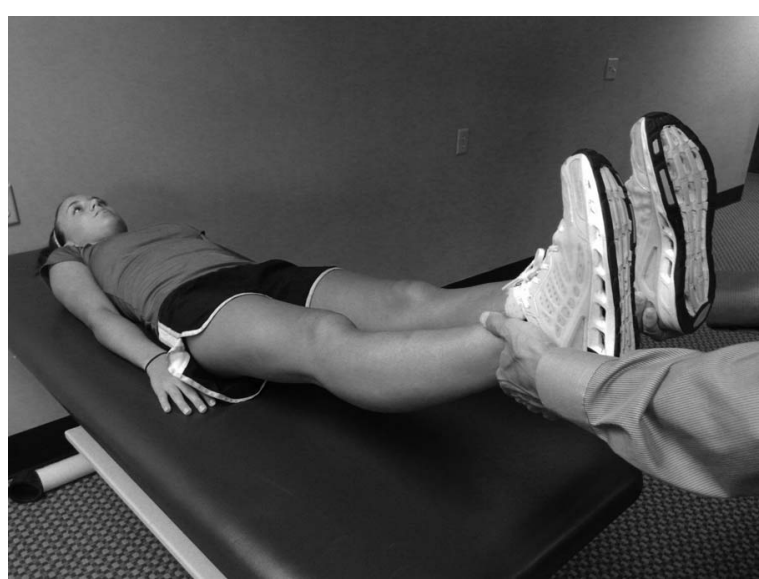

Figure 10 Bilateral adductor test.

Diagnostic accuracy: SN 30\%, SP 91\%, +LR 3.3 and -LR $0.66 .^{32}$

Special note: Force is applied at the ankle with the knee straight.

Background: As with the other sports related chronic groin tests, adduction contraction elicits stress across the common origin of the adductor muscles on the pubic symphysis region.

\section{Squeeze test}

Patient position: Supine with bilateral hips flexed $45^{\circ}$ and knees flexed $90^{\circ}$ so that bilateral feet are flat on table. ${ }^{32}$

Clinician position: Standing at patient's bilateral knees, placing fist between knees.

Movement: Patient is asked to contract maximally both adductor muscles simultaneously to 'squeeze the fist' effectively (figure 9).

Assessment: Reproduction of patient's concordant pain is considered a positive test.

Diagnostic accuracy: SN 43\%, SP 91\%, +LR 4.8 and -LR $0.63 .^{32}$

Special note: Monitor for patient compensation of lower trunk rotation.

Background: As with the other sports-related chronic groin tests, adduction contraction elicits stress across the common origin of the adductor muscles on the pubic symphysis region.

\section{Bilateral adductor test}

Patient position: Patient is supine with bilateral legs extended. ${ }^{32}$

Clinician position: Standing at patient's bilateral feet, directly facing patient.

Movement: Patient is asked to contract maximally both adductor muscles simultaneously, thereby attempting to bring bilateral legs together (figure 10).

Assessment: Reproduction of patient's concordant pain is considered a positive test.

Diagnostic accuracy: SN 54\%, SP 93\%, +LR 7.7 and -LR $0.49 .^{32}$

Special note: Monitor for compensations of patient grabbing onto table and/or bending knees.

Background: As with the other sports related chronic groin tests, adduction contraction elicits stress across the common origin of the adductor muscles on the pubic symphysis region.

Commentary on sports related chronic groin pain tests

The bilateral adductor test was the most diagnostic of these tests, with the potential to alter post-test probability to a moderate degree. ${ }^{8}$ The other two tests, single adductor test and the squeeze test, also have greater capability as a diagnostic versus screening test. Both of these tests can alter posttest probability to a small degree.

\section{DISCUSSION}

Clinical testing of the hip is not nearly as comprehensively investigated as other body part regions such as the shoulder and the knee. As such the collection of clinical tests available to diagnostic clinicians is somewhat underwhelming in context. This may be one of the reasons patients with hip problems frequently undergo inappropriate imaging and inappropriate treatments, and have a delayed proper diagnosis. ${ }^{34}$

This manuscript focuses on the detailed execution of 10 index tests for the hip joint. Each is described in sufficient detail using the sentinel reference when available. The tests were selected from the recent systematic review and meta-analysis from Reiman et al, ${ }^{8}$ because to our knowledge, this study is the most comprehensive investigation of the diagnostic accuracy of the hip joint.

And added benefit of a description paper is that the execution of the tests can be standardised across future studies. When possible, we used the sentinel references for the description of each index test. Variation in test performance is a form of bias that has been recognised in the original QUADAS ${ }^{33}$ and QUADAS $\mathrm{II}^{34}$ quality assessment instruments. Consistent use of these tests in future well designed studies should more efficiently determine the true value of these tests during examination of the hip joint.

\section{What is already known on this topic}

- Hip joint examination is becoming increasingly popular with improving technology regarding examination and treatment.

- Multiple studies have been describing various hip physical examination (HPE) tests. Currently, reviews have only focused on labral pathology. Reiman et $a l^{8}$ in the British Journal of Sports Medicine is the only systematic review to examine the clinical utility of HPE tests for all hip pathology.

- Description of the various HPE tests is quite variable dependent on the study.

\section{What this study adds}

This study describes the correct performance of the best HPE tests as described by Reiman et $a l^{8}$ The variable description in multiple studies for similar tests requires a detailed description (and photograph) of the test procedure for each relevant hip pathology.

Acknowledgements The authors would like to thank Carly Reiman for participating in the photographs.

Contributors MPR provided idea, design, writing, review of manuscript and overall content of material; Mather and Cook provided writing, review and overall content of manuscript.

Competing interests None.

Provenance and peer review 


\section{REFERENCES}

1 Haviv B, Burg A, Velkes $S$, et al. Trends in femoroacetabular impingement research over 11 years. Orthopedics 2011;34:353.

2 Ganz R, Parvizi J, Beck M, et al. Femoroacetabular impingement: a cause for osteoarthritis of the hip. Clin Orthop Relat Res 2003;417:112-20.

3 Burnett RS, Della Rocca GJ, Prather $\mathrm{H}$, et al. Clinical presentation of patients with tears of the acetabular labrum. J Bone Joint Surg Am 2006;88:1448-57.

4 Nunley RM, Prather $\mathrm{H}$, Hunt $\mathrm{D}$, et al. Clinical presentation of symptomatic acetabular dysplasia in skeletally mature patients. J Bone Joint Surg Am 2011;93(Suppl 2):17-21.

5 Philippon MJ, Weiss DR, Kuppersmith DA, et al. Arthroscopic labral repair and treatment of femoroacetabular impingement in professional hockey players. Am J Sports Med 2010;38:99-104.

$6 \mathrm{McC}$ arthy J, Noble P, Aluisio FV, et al. Anatomy, pathologic features, and treatment of acetabular labral tears. Clin Orthop Relat Res 2003;406:38-47.

7 Byrd JW. Snapping hip. Oper Tech Sports Med 2005;13:303-8.

8 Reiman MP, Goode AP, Hegedus EJ, et al. Diagnostic accuracy of clinical tests of the hip: a systematic review with meta-analysis. Br J Sports Med 2013;47: 893-902.

9 Youdas JW, Madson TJ, Hollman JH. Usefulness of the Trendelenburg test for identification of patients with hip joint osteoarthritis. Physiotherapy [Validation Studies] 2010;26:184-94.

10 Lequesne $M$, Mathieu $P$, Vuillemin-Bodaghi V, et al. Gluteal tendinopathy in refractory greater trochanter pain syndrome: diagnostic value of two clinical tests. Arthritis Rheum 2008;59:241-6.

11 Hardcastle $\mathrm{P}$, Nade $\mathrm{S}$. The significance of the Trendelenburg test. J Bone Joint Surg Br 1985;67:741-6.

12 Altman $\mathrm{R}$, Alarcon G, Appelrouth $\mathrm{D}$, et al. The American College of Rheumatology criteria for the classification and reporting of osteoarthritis of the hip. Arthritis Rheum 1991;34:505-14.

13 Birrell F, Croft P, Cooper C, et al. Predicting radiographic hip osteoarthritis from range of movement. Rheumatology (Oxford) [Multicenter Study Research Support, Non-U.S. Gov't]. 2001:40:506-12.

14 Bird PA, Oakley SP, Shnier R, et al. Prospective evaluation of magnetic resonance imaging and physical examination findings in patients with greater trochanteric pain syndrome. Arthritis Rheum 2001;44:2138-45.

15 Woodley SJ, Nicholson HD, Livingstone V, et al. Lateral hip pain: findings from magnetic resonance imaging and clinical examination. J Orthop Sports Phys Ther [Research Support, Non-U.S. Gov't] 2008;38:313-28.

16 Troelsen A, Mechlenburg I, Gelineck J, et al. What is the role of clinical tests and ultrasound in acetabular labral tear diagnostics? Acta Orthop 2009;80:314-18.

17 Beaule PE, Zaragoza E, Motamedi K, et al. Three-dimensional computed tomography of the hip in the assessment of femoroacetabular impingement. J Orthop Res 2005;23:1286-92.
18 Leunig $\mathrm{M}$, Werlen $\mathrm{S}$, Ungersbock $\mathrm{A}$, et al. Evaluation of the acetabular labrum by MR arthrography. J Bone Joint Surg Br 1997;79:230-4.

19 Keeney JA, Peelle MW, Jackson J, et al. Magnetic resonance arthrography versus arthroscopy in the evaluation of articular hip pathology. Clin Orthop Relat Res 2004 (429):163-9.

20 Sink EL, Gralla J, Ryba A, et al. Clinical presentation of femoroacetabular impingement in adolescents. J Pediatr Orthop 2008;28:806-11.

21 Chan YS, Lien LC, Hsu HL, et al. Evaluating hip labral tears using magnetic resonance arthrography: a prospective study comparing hip arthroscopy and magnetic resonance arthrography diagnosis. Arthroscopy 2005;21:1250.

22 Hase T, Ueo T. Acetabular labral tear: arthroscopic diagnosis and treatment. Arthroscopy 1999;15:138-41.

23 Petersilge CA, Haque MA, Petersilge WJ, et al. Acetabular labral tears: evaluation with MR arthrography. Radiology 1996;200:231-5.

24 McCarthy JC, Busconi B. The role of hip arthroscopy in the diagnosis and treatment of hip disease. Orthopedics 1995;18:753-6.

25 Lewis CL, Sahrmann SA, Moran DW. Anterior hip joint force increases with hip extension, decreased gluteal force, or decreased iliopsoas force. J Biomech 2007:40:3725-31.

26 Adams SL, Yarnold PR. Clinical use of the patellar-pubic percussion sign in hip trauma. Am J Emerg Med 1997;15:173-5.

27 Bache JB, Cross AB. The Barford test. A useful diagnostic sign in fractures of the femoral neck. Practitioner 1984;228:305-8.

28 Tiru M, Goh SH, Low BY. Use of percussion as a screening tool in the diagnosis of occult hip fractures. Singapore Med J 2002;43:467-9.

29 Johnson AW, Weiss CB Jr, Wheeler DL. Stress fractures of the femoral shaft in athletes-more common than expected. A new clinical test. Am J Sports Med 1994:22:248-56.

30 Kang L, Belcher D, Hulstyn MJ. Stress fractures of the femoral shaft in women's college lacrosse: a report of seven cases and a review of the literature. $\mathrm{Br}$ J Sports Med 2005;39:902-6.

31 Moore MB. The use of a tuning fork and stethoscope to identify fractures. J Athl Train 2009:44:272-4

32 Verrall GM, Slavotinek JP, Barnes PG, et al. Description of pain provocation tests used for the diagnosis of sports-related chronic groin pain: relationship of tests to defined clinical (pain and tenderness) and MRI (pubic bone marrow oedema) criteria. Scand J Med Sci Sports 2005;15:36-42.

33 Whiting P, Rutjes AW, Reitsma JB, et al. The development of QUADAS: a tool for the quality assessment of studies of diagnostic accuracy included in systematic reviews. BMC Med Res Methodol 2003;3:25.

34 Whiting PF, Rutjes AW, Westwood ME, et al. QUADAS-2: a revised tool for the quality assessment of diagnostic accuracy studies. Ann Intern Med2011;155:529-36. 